isid/ms/2008/02

March 31, 2008

http://www.isid.ac.in/statmath/eprints

\title{
Super Efficient Frequency Estimation
}

\author{
Debasis Kundu \\ ZHIDONG BAI \\ SWAGATA NANDI
}

LI BAI

Indian Statistical Institute, Delhi Centre

7, SJSS Marg, New Delhi-110 016, India 



\title{
Super Efficient Frequency Estimation
}

\author{
Debasis Kundu ${ }^{1}$, Zhidong Bai ${ }^{2}$, Swagata Nandi ${ }^{3}$ and Li Bai ${ }^{4}$ \\ ${ }^{1}$ Department of Mathematics and Statistics \\ Indian Institute of Technology KanPuR \\ Pin 208016, INDIA \\ KUNDU@IITK.AC.IN \\ ${ }^{2}$ Department of Statistics and Applied Probability \\ Block S16, Level 7, 6 Science Drive 2, Faculty of Science, \\ The National University of Singapore \\ SINGAPORE 117546 \\ STABAIZD@NUS.EDU.SG \\ ${ }^{3}$ Theoretical Statistics and Mathematics Unit \\ Indian Statistical Institute \\ 7, S.J.S. Sansanwal Marg \\ New Delhi - 110016, India \\ NANDI@ISID.AC.IN \\ ${ }^{4}$ Department of Electrical and Computer Engineering \\ College of Engineering \\ Temple University, USA \\ LBAI@TEMPLE.EDU
}

\begin{abstract}
In this paper we propose a modified Newton-Raphson method to obtain super efficient frequency estimators of sinusoidal signals in presence of stationary noise. It is observed that if we start from an initial estimator with convergence rate $O_{p}\left(n^{-1}\right)$ and use the NewtonRaphson algorithm with proper step factor modification, then it produces super efficient frequency estimator in the sense it has asymptotic variance which is lower than the asymptotic variance of the least squares estimator. The proposed frequency estimator is consistent and have the optimum rate of convergence, namely $O_{p}\left(n^{-\frac{3}{2}}\right)$, which is same as the least squares estimator. Monte Carlo simulations are performed to observe the performances of the proposed estimator for different sample sizes and for different models. The results are
\end{abstract}


quite satisfactory. One real data set has been analyzed for illustrative purpose.

KEYWORDS: Sinusoidal signals; least squares estimators; asymptotic distributions; modified Newtod-Raphson algorithm.

2000 Mathematics Subject Classification: 62J02; 62E20; 62 C05.

Corresponding AUthor: Swagata Nandi, Phone: 91-11-41493931, Fax: 91-11-41493981, e-mail: nandi@isid.ac.in.

\section{INTRODUCTION}

In this paper we consider the problem of estimating the unknown parameters of the following sinusoidal signals observed in presence of stationary noise. The model can be described as follows;

$$
y(t)=A \cos (\omega t)+B \sin (\omega t)+Z(t) ; \quad t=1, \ldots, n .
$$

Here $A, B$ are unknown amplitudes and $\omega$ is known as the frequency. The amplitudes are unknown and they belong to $(-\infty, \infty)$ and the frequency $\omega$ is also unknown and it lies in $(0, \pi)$. The additive noise $\{Z(t)\}$ is a sequence of stationary random variables with mean zero and finite variance and it satisfies the following Assumption 1.

Assumption 1: The random variable $\{Z(t)\}$ has the following linear structure

$$
Z(t)=\sum_{k=-\infty}^{\infty} a(k) e(t-k),
$$

where $\{e(t)\}$ is a sequence of independent and identically distributed (i.i.d.) random variables with mean zero and finite variance $\sigma^{2}$. The real valued sequence $\{a(t)\}$ satisfies the following condition

$$
\sum_{k=-\infty}^{\infty}|a(k)|<\infty
$$


The main problem is to estimate the unknown amplitudes and frequency, given a sample of size $n$, namely $\{y(1), \ldots, y(n)\}$. It is known, see Walker [16], that if the frequency (non-linear parameter) is estimated with the convergence rate $O_{p}\left(n^{-\frac{3}{2}}\right)$, here $O_{p}\left(n^{-\delta}\right)$ means $n^{\delta} O_{p}\left(n^{-\delta}\right)$ is bounded in probability, then the amplitudes (linear parameters) can be estimated efficiently with the convergence rate $O_{p}\left(n^{-\frac{1}{2}}\right)$. In this paper we mainly address the efficient frequency estimator, which has the rate of convergence $O_{p}\left(n^{-\frac{3}{2}}\right)$ but it has the asymptotic variance smaller than the asymptotic variance of the least squares estimator (LSE), so we call it as super efficient frequency estimator.

This is an important, age old and well known to be a difficult problem. This particular model or some of its generalizations have been used in more or less all areas of science and engineering. Fisher first discussed this problem in 1929, see Fisher [5]. Since then several articles have been published establishing the theoretical properties and developing different numerical algorithms, see for example Hannan [6], Walker [16], Bai et al. [2], Smyth [13], the list of references by Stoica [15], Bai et al. [1], Nandi and Kundu [8], Song and Li [14] for some recent developments.

There are two important aspects of this problem. The first one is the properties of the LSEs of the unknown parameters. Interestingly, unlike other non-linear regression model, the rate of convergence of the LSE of the frequency is $O_{p}\left(n^{-\frac{3}{2}}\right)$, but the rate of convergence of the LSEs of the amplitudes is $O_{p}\left(n^{-\frac{1}{2}}\right)$, see for example Hannan [6] or Walker [16]. The periodogram maximizers without the restriction on the Fourier frequencies ( $\omega$ is Fourier frequency if $\omega=\frac{2 \pi k}{n}$, for some integer $k$ in $\left.\left(1,2, \ldots,\left[\frac{N}{2}\right]\right)\right)$ also provide the optimum convergence rate $O_{p}\left(n^{-\frac{3}{2}}\right)$. Moreover, it is known that the LSE of the frequency and the periodogram maximizer are asymptotically equivalent.

The second important aspect of this particular problem is to find the LSEs of the unknown parameters. It is known, see Rice and Rosenblatt [11], that the least squares surface is highly non-linear. The period of $y(t)$ becomes small as $n$ becomes large and hence the oscillation of the 
sum of the residual squares increases. Thus there will be too many local minimizers. Therefore, very good (close enough to the true values) initial estimates are needed for any iterative process to converge. Moreover, the periodogram maximizer over Fourier frequencies does not work very well as the convergence rate is only $O_{p}\left(n^{-1}\right)$, see Rice and Rosenblatt [11], whereas the initial estimate with convergence rate $O_{p}\left(n^{-1-\delta}\right)(\delta>0)$ is needed for most of the iterative process to work satisfactorily. It is well known, see Smyth [13], that the standard Newton-Raphson algorithm can not be used in this case, because often it does not converge or it may converge to a local optimum rather than the global optimum. Several techniques are available in the literature for example Pisarenko [9], Chan et al. [4] which attempt to find computationally efficient algorithm for estimating the frequency, but these procedures produce estimates having convergence rate $O_{p}\left(n^{-\frac{1}{2}}\right)$. Recently, Bai et al. [1], see also Nandi and Kundu [8], proposed an efficient estimation procedure of the frequency, which has the optimum convergence rate $O_{p}\left(n^{-\frac{3}{2}}\right)$ and it convergences within a fixed number of iterations.

The main aim of this paper is to provide a modified Newton-Raphson algorithm for estimating the unknown frequency with the optimum rate of convergence. It is known that the LSE of the frequency can be obtained by maximizing a quadratic form involving the data (see Sec. 2.1) and the unknown frequency. We use the Newton-Raphson algorithm with a step factor modification. Although, in general the Newton-Raphson algorithm does not work well for this problem, but with the step factor modification it works very well. It is observed that if we start the iterative process from an estimator which has the convergence rate $O_{p}\left(n^{-1}\right)$, then it produces super efficient frequency estimator, with the convergence rate $O_{p}\left(n^{-\frac{3}{2}}\right)$ and having smaller asymptotic variance than the LSE. The results can be easily extended to the multiple sinusoids using sequential estimation method.

The rest of the paper is organized as follows. In section 2, we briefly describe the least squares and approximate least squares procedures for estimating the frequency. In Section 3, we provide the proposed modified Newton-Raphson algorithm. In Section 4, we consider the 
case when more than one frequency is present. Simulation results are presented in Section 5. For illustrative purpose, one data set has been analyzed in Section 6, and finally we conclude the paper in Section 7. All the necessary proofs are presented in the Appendix.

\section{Estimating the Unknown Parameters}

In the literature there are two well known methods available for estimating the unknown frequency of the model (1). One is the standard least squares method and the other is the periodogram maximizer or known as the approximate least squares method. We will briefly describe them here for further development.

\subsection{Least Squares Method}

The LSEs of $A, B$ and $\omega$ can be obtained by minimizing

$$
Q(A, B, \omega)=\sum_{t=1}^{n}(y(t)-A \cos (\omega t)-B \sin (\omega t))^{2}
$$

with respect to $A, B$ and $\omega$. We do it in two stages, first we minimize with respect to (w.r.t.) $A$ and $B$ for fixed $\omega$ and then minimize w.r.t. $\omega$. If we use the following notation

$$
X=\left[\begin{array}{cc}
\cos (\omega) & \sin (\omega) \\
\vdots & \vdots \\
\cos (n \omega) & \sin (n \omega)
\end{array}\right], \quad Y=(y(1), \ldots, y(n))^{T}
$$

then minimizing $Q(A, B, \omega)$ w.r.t. $A, B$ and $\omega$ boils down to maximize $R(\omega)$ w.r.t. $\omega$, where

$$
R(\omega)=Y^{T} X\left(X^{T} X\right)^{-1} X^{T} Y
$$

The matrix $X$ depends on $\omega$, but we do not make it explicit unless necessary. If $\widehat{\omega}$, maximizes (6), then the estimators of $A$ and $B$ can be obtained using the separable regression technique 
of Richards [12] as

$$
(\widehat{A} \widehat{B})^{T}=\left(X(\widehat{\omega})^{T} X(\widehat{\omega})\right)^{-1} X(\widehat{\omega})^{T} Y
$$

Most of the numerically efficient algorithms, for example the methods proposed by Bresler and Macovski [3], Kumaresan et al.[7], and Smyth [13] attempt to maximize (6), which naturally saves computational times as the optimization takes place in one dimension.

\subsection{Approximate Least Squares Method}

The approximate LSE of the frequency can be obtained by maximizing the periodogram function $I(\omega)$, w.r.t. $\omega \in(0, \pi)$, when $I(\omega)$ is defined as follows;

$$
I(\omega)=\left|\frac{1}{n} \sum_{t=1}^{n} y(t) e^{-i \omega t}\right|^{2} .
$$

Once the periodogram maximizer or the approximate LSE of $\omega$, say $\widehat{\widehat{\omega}}$ is obtained, the estimators of the amplitudes can easily be obtained as before. It is known that, see Hannan [6], Walker [16] that both $\widehat{\omega}$ and $\widehat{\widehat{\omega}}$ are consistent estimators of $\omega$ and they are asymptotically equivalent, with the following asymptotic distributions;

$$
\begin{aligned}
& n^{\frac{3}{2}}(\widehat{\omega}-\omega) \stackrel{d}{\longrightarrow} \mathcal{N}\left(0, \frac{24 \sigma^{2} c}{A^{2}+B^{2}}\right), \\
& n^{\frac{3}{2}}(\widehat{\widehat{\omega}}-\omega) \stackrel{d}{\longrightarrow} \mathcal{N}\left(0, \frac{24 \sigma^{2} c}{A^{2}+B^{2}}\right),
\end{aligned}
$$

where

$$
c=\left|\sum_{k=-\infty}^{\infty} a(k) e^{i k \omega}\right|^{2}=\left|\sum_{k=-\infty}^{\infty} a(k) \cos (k \omega)\right|^{2}+\left|\sum_{k=-\infty}^{\infty} a(k) \sin (k \omega)\right|^{2},
$$

' $\stackrel{d}{\longrightarrow}$ ' means convergence in distribution and $\mathcal{N}(a, b)$ means normal distribution with mean $a$ and variance $b$. In next section, we modify the Newton-Rhapson Algorithm using the set-up of this section. 


\section{Modified Newton-Raphson Algorithm}

Our aim is to maximize $R(\omega)$ w.r.t. $\omega$ and we propose the modified Newton-Raphson algorithm as follows. If $\widehat{\omega}_{0}$ is an initial estimate of $\omega$, then the next iterate $\widehat{\omega}_{1}$ is obtained as

$$
\widehat{\omega}_{1}=\widehat{\omega}_{0}-\frac{1}{4} \times \frac{R^{\prime}\left(\widehat{\omega}_{0}\right)}{R^{\prime \prime}\left(\widehat{\omega}_{0}\right)}
$$

similarly, $\widehat{\omega}_{2}$ can be obtained from $\widehat{\omega}_{1}$ by replacing $\widehat{\omega}_{0}$ with $\widehat{\omega}_{1}$ in $(12)$. Here $R^{\prime}\left(\widehat{\omega}_{0}\right)$ and $R^{\prime \prime}\left(\widehat{\omega}_{0}\right)$ are the first and second derivatives of $R(\omega)$ respectively, evaluated as $\omega=\widehat{\omega}_{0}$. Note that the iterative process proposed in (12) is the Newton-Raphson algorithm with the step factor modification $\frac{1}{4}$. The standard Newton-Raphson algorithm in this case is same as (12), without the factor $\frac{1}{4}$. So we update the estimator with four times smaller step factor. It is well known that the standard Newton-Raphson algorithm does not work well for this model. Most of the times it does not even converge. Interestingly, with the step factor modification $\frac{1}{4}$, the algorithm works very well and it produces super efficient frequency estimator. We shall see in section 5, that this modification prevents the procedure to converge to a local maxima or diverge. The reason to choose the factor $\frac{1}{4}$ will be clear from Theorem 1 whose proof is provided in the Appendix.

Theorem 1. Suppose $\widehat{\omega}_{0}$ is an estimate of $\omega$ and $\widehat{\omega}_{0}-\omega=O_{p}\left(n^{-1-\delta}\right)$, where $\delta \in\left(0, \frac{1}{2}\right]$. If

$$
\widehat{\omega}=\widehat{\omega}_{0}-\frac{1}{4} \times \frac{R^{\prime}\left(\widehat{\omega}_{0}\right)}{R^{\prime \prime}\left(\widehat{\omega}_{0}\right)}
$$

then

(a) $\widehat{\omega}-\omega=O_{p}\left(n^{-1-3 \delta}\right) \quad$ if $\delta \leq \frac{1}{6}$

(b) $n^{\frac{3}{2}}(\widehat{\omega}-\omega) \stackrel{d}{\longrightarrow} N\left(0, \frac{6 \sigma^{2} c}{A^{2}+B^{2}}\right) \quad$ if $\delta>\frac{1}{6}$,

here $c$ is same as defined in (11). 
Implementation: We start the process with an initial estimator with the convergence rate $O_{p}\left(n^{-1}\right)$ and a fraction of the available data points. Note that getting an initial estimator of the frequency with the convergence rate $O_{p}\left(n^{-1}\right)$ is not difficult. For example, the argument maximum of the periodogram function $I(\omega)$ as defined in (8) or the function $R(\omega)$ as defined in (6), over Fourier frequencies will produce an initial estimator with the required convergence rate.

Now based on part (a) of Theorem 1, and using similar ideas as of Bai et al. [1] or Nandi and Kundu [8], from an estimator with the convergence rate $O_{p}\left(n^{-1}\right)$, it is possible to get an estimator with the convergence rate $O_{p}\left(n^{-1-\delta}\right)$ for $\delta>\frac{1}{6}$. Therefore, using part (b) of Theorem 1 , it follows that the iterative process (12) will produce frequency estimate whose asymptotic variance is lower than the asymptotic variance of the least squares estimate.

We propose the following algorithm. Find the argument maximum of $R(\omega)$ at the Fourier frequency which has the convergence rate $O_{p}\left(n^{-1}\right)$. Suppose it is $\widehat{\omega}_{0}$. Take $n_{1}=n^{\frac{6}{7}}$, and consider

$$
\widehat{\omega}_{1}=\widehat{\omega}_{0}-\frac{1}{4} \times \frac{R^{\prime}\left(\widehat{\omega}_{0}\right)}{R^{\prime \prime}\left(\widehat{\omega}_{0}\right)}
$$

Here $R^{\prime}\left(\widehat{\omega}_{0}\right)$ and $R^{\prime \prime}\left(\widehat{\omega}_{0}\right)$ are computed based on sample size $n_{1}$. Since, $\widehat{\omega}_{0}-\omega=O_{p}\left(n^{-1}\right)$ and $n_{1}=n^{\frac{6}{7}}$, therefore, $n^{-1}=n_{1}^{-\frac{7}{6}}=n_{1}^{-1-\frac{1}{6}}$ and $\widehat{\omega}_{0}-\omega=O_{p}\left(n_{1}^{-1-\frac{1}{6}}\right)$. Now based on the result (a) of Theorem 1, we have

$$
\widehat{\omega}_{1}-\omega=O_{p}\left(n_{1}^{-1-\frac{1}{2}}\right)=O_{p}\left(n^{-\frac{3}{2} \times \frac{6}{7}}\right)=O_{p}\left(n^{-\frac{9}{7}}\right)=O_{p}\left(n^{-1-\frac{2}{7}}\right) .
$$

Since $\frac{2}{7}>\frac{1}{6}$, therefore, we will be able to apply part (b) of Theorem 1 repeatedly to produce super efficient frequency estimator. That is, using the same sample size $n$, repeat

$$
\widehat{\omega}_{k+1}=\widehat{\omega}_{k}-\frac{1}{4} \times \frac{R^{\prime}\left(\widehat{\omega}_{k}\right)}{R^{\prime \prime}\left(\widehat{\omega}_{k}\right)}
$$

with $k=1,2, \ldots$ until a suitable stopping criterion is satisfied.

Remark 1. In equation (7), let us write $(\widehat{A}, \widehat{B})^{T}=(A(\widehat{\omega}), B(\widehat{\omega}))^{T}$. Now expanding $A(\widehat{\omega})$ at 
$\omega^{0}$ by Taylor series, we have

$$
A(\widehat{\omega})-A\left(\omega^{0}\right)=\left(\widehat{\omega}-\omega^{0}\right) A^{\prime}(\bar{\omega})+o\left(n^{2}\right)
$$

where $A^{\prime}(\bar{\omega})$ is the first derivative of $A(\omega)$ at $\bar{\omega}, \bar{\omega}$ is a point between $\widehat{\omega}$ and $\omega^{0}$. Here $\widehat{\omega}$ can be either the least squares estimator or the estimator obtained by modified Newton Raphson method. Now comparing the variances (asymptotic) of the two estimators of $\omega$, it is clear that the asymptotic variance of the corresponding estimator of $A$ is four times less than that of the LSE. The same is true for the estimator of $B$.

Remark 2. It is well-known that the Newton-Raphson algorithm in its original form does not work well for sinusoidal model. But interestingly, with the step factor modification, it not only provides estimator with the same rate of convergence as the LSE but has asymptotic variance less than that of the LSE.

Remark 3. The frequency parameter of the sinusoidal model with stationary error has the

Cramer-Rao Lower Bound (CRLB) as $\frac{24 \sigma^{2} c}{A^{2}+B^{2}}$. In this paper, we propose an estimator obtained iteratively which has the asymptotic variance lower than the CRLB.

So we think that the novelty of this work lies on the fact that it modifies the NewtonRaphson method in such a way that it produces super-efficient estimator in case of sinusoidal model, usually which may not even converge.

\section{Multiple Sinusoids}

In this section we consider estimating the unknown parameters for multiple sinusoidal model, which is the usual case in practice. We consider the following model;

$$
y(t)=\sum_{j=1}^{p}\left[A_{j} \cos \left(\omega_{j} t\right)+B_{j} \sin \left(\omega_{j} t\right)\right]+Z(t) ; \quad t=1, \ldots, n .
$$


Here $A_{j}$ 's and $B_{j}$ 's are unknown amplitudes, can take any arbitrary real value and $\omega_{j}$ 's are unknown frequencies and $\in(0, \pi)$. It is assumed that $\omega_{j}$ 's are distinct and the number of components $p$ is known in advance. The error random variable $Z(t)$ satisfies the same assumption as before. For identifiably purposes, it is assumed that

$$
A_{1}^{2}+B_{1}^{2} \geq \ldots \geq A_{p}^{2}+B_{p}^{2}
$$

moreover if for any $1 \leq k \leq p-1, A_{k}^{2}+B_{k}^{2}=A_{k+1}^{2}+B_{k+1}^{2}$, then it is assumed $\omega_{k}>\omega_{k+1}$.

In this section also we mainly consider the estimation of the unknown frequencies $\omega_{1}, \ldots, \omega_{p}$ from the given sample $\{y(1), \ldots, y(n)\}$, because once we have estimates of the frequencies, the estimates of the amplitudes can be obtained using the separable regression technique of Richards [12] as before. We use the sequential estimation procedure to estimate the unknown parameters if more than one frequency is present. The procedure can be described as follows. If $\widehat{\omega}_{0}$ is the maximizer of $R(\omega)$ over Fourier frequencies, then estimate $\omega_{1}$ by $\widehat{\omega}_{1}$ using the algorithm described in the previous section. It can be shown that

$$
n^{\frac{3}{2}}\left(\widehat{\omega}_{1}-\omega_{1}\right) \stackrel{d}{\longrightarrow} \mathcal{N}\left(0, \frac{6 \sigma^{2} c}{A_{1}^{2}+B_{1}^{2}}\right) .
$$

Now using (7) estimate $\widehat{A}_{1}$ and $\widehat{B}_{1}$ replacing $\widehat{\omega}$ by $\widehat{\omega}_{1}$. Now consider the following modified data taking out the effect of the frequency $\omega_{1}$ as follows;

$$
\tilde{y}(t)=y(t)-\widehat{A}_{1} \cos \left(\widehat{\omega}_{1} t\right)-\widehat{B}_{1} \sin \left(\widehat{\omega}_{1} t\right), \quad t=1, \ldots, n
$$

Now applying the same procedure on the modified data $\{\tilde{y}(1), \ldots, \tilde{y}(n)\}$ we estimate $\omega_{2}$. Repeating the procedure $p$ times we can obtain super efficient estimates of the frequencies. The method automatically gives an estimate of the number of component, $p$. If we repeat the above procedure say $q$ times and we are left with a pure random sequence, then $q$ is an estimate of $p$. This has been considered in Data Analysis section to analyze one real dataset. 


\section{NumERICAL EXPERIMENT}

In this section, we present some experimental results to observe how the proposed algorithm performs for different sample sizes and for different models. We use the random deviate generator RAN2 of Press et al. [10]. All the programs are written in FORTRAN and they can be obtained from the corresponding author on request. We consider the following two models;

- Model 1: $y(t)=2 \cos \left(0.5 t+\frac{\pi}{4}\right)+Z(t)$.

- Model 2: $y(t)=2 \cos \left(1.5 t+\frac{\pi}{3}\right)+2 \cos \left(0.5 t+\frac{\pi}{4}\right)+Z(t)$.

The error random variable $Z(t)$ is an autoregressive process on order one, of the form

$$
Z(t)=.5 \quad Z(t-1)+e(t)
$$

where $\{e(t)\}$ is a sequence of i.i.d. Gaussian random variables with mean zero and variance $\sigma^{2}$. We consider different sample sizes and different error variances, namely $n=100,200,300$, 400, 500, 1000 and $\sigma=0.25,0.50,0.75,1.00,1.50$.

In each case we generate the sample from the given model and compute the initial estimate from $R(\omega)$ as discussed in section 3. We compute the final estimate $\widehat{\omega}$ for Model 1 , using the iterative process proposed in (12). We stop the iterative process if the absolute difference between the consecutive iterates is less than $\frac{\sigma^{2}}{n^{3}}$. It is observed that in most of the cases, the iteration converges within 8-10 iterations. In case on Model 2, we repeat the process again after removing the effect of the first frequency to estimate the parameters of second component. We replicate the process 1000 times and compute the average estimates (AEs) and their mean squared errors (MSEs). For comparison purposes we report the asymptotic variances of the least squares estimators (ASV) as given in (9) as well as the proposed asymptotic variance (ASVP) as provided in part (b) of Theorem 1. We report results for frequencies of both the models considered here. The results for Model 1 are reported in Tables 2 and for Model 2 in 
Tables 3 and 4. Following remark 1, we expect that the MSEs of $\widehat{A}$ and $\widehat{B}$ may be smaller than the corresponding CRLBs. So we have reported the results of $A$ in case of Model 1 in Table 5. Similar trend has been observed in other cases, so they are not reported here.

Some of the points are quite clear from Tables 2-5. It is observed that as the sample size increases or the variance decreases the MSEs and the biases decrease for different parameter estimators of both the models. It verifies the consistency properties of the proposed estimates. Moreover, the MSEs of the proposed estimates are usually smaller than the asymptotic variances of the LSEs and we expect them that way following Theorem 1. This is observed in case of frequencies as well as the linear parameters (Remark 1). So the improvement is achievable in practice. Since the implementation of the proposed iterative procedure is quite simple, therefore, it can be used quite effectively for data analysis purpose.

\section{Data Analysis}

In this section we use the multiple sinusoidal model to study a periodic data. The data represent the rainfall of the homogeneous Indian monthly rainfall data. The data were collected from the website (http://www.tropmet.res.in/) of the Indian Institute of Tropical Meteorology and presented in Fig. 1. The original data set contains 1608 data points. To understand how good the fit is we have considered a subset of 200 data points to demonstrate. Actual fitting was done for the whole data set. The initial estimates are obtained by maximizing the function $R(\omega)$ over the Fourier frequencies. After obtaining the estimate of $\omega_{1}$, we use the proposed method and obtain the final estimate of $\omega_{1}$. The stopping criterion used here is same as the one considered in simulation study. We stop the iterative process if the absolute difference between two iterates is less $n^{-3}$ which is approximately $2.5 \times 10^{-10}$ for the given sample size. Then calculate the corresponding amplitudes and remove the effect of $\omega_{1}$ from $y(t)$. Let us denote the resulting modified $y(t)$ as $\tilde{y}(t)$ (as in equation (16)). Now assuming $\tilde{y}(t)$ as the data, 
Table 1: Parameter estimates of the homogeneous Indian rainfall data

\begin{tabular}{|c|c|c|}
\hline$A$ & $B$ & $\omega$ \\
\hline-0.773770034 & -0.810867786 & 0.523574177 \\
0.0699929297 & -0.207292303 & 1.57058741 \\
0.0497323982 & 0.091640681 & 2.09340777 \\
0.0248339158 & 0.640113711 & 1.04710327 \\
0.00398120238 & -0.107113108 & 2.61765981 \\
\hline
\end{tabular}

we repeat the same procedure and subsequently, maximizing $R(\omega)$ step by step, we estimate all the frequencies present in the data and continue the process till all significant periodic components are estimated and a pure random quantity is left. We observe that there are five significant frequencies present. The random part can be approximated as a stationary process which is according to the model assumption. The data were mean-corrected and scaled by the square root of the estimated variance to estimate the parameters. Then the above stopping criterion has been used. The corresponding estimates are present in Table 1. The fitted values are calculated considering the scale and the mean and are given in Fig. 2. For better understanding and comparison, we have plotted the observed values also in the same Figure. The fitted values match quite well with the observed $y(t)$.

\section{Conclusions}

In this paper we have considered the estimation of the unknown parameters of a classical model, namely the sum of sinusoidal model. We have mainly discussed the effective estimation of the unknown frequencies. Although, it has been criticized heavily in the literature against the use of Newton-Raphson method for computing the least squares estimators of the unknown frequencies 


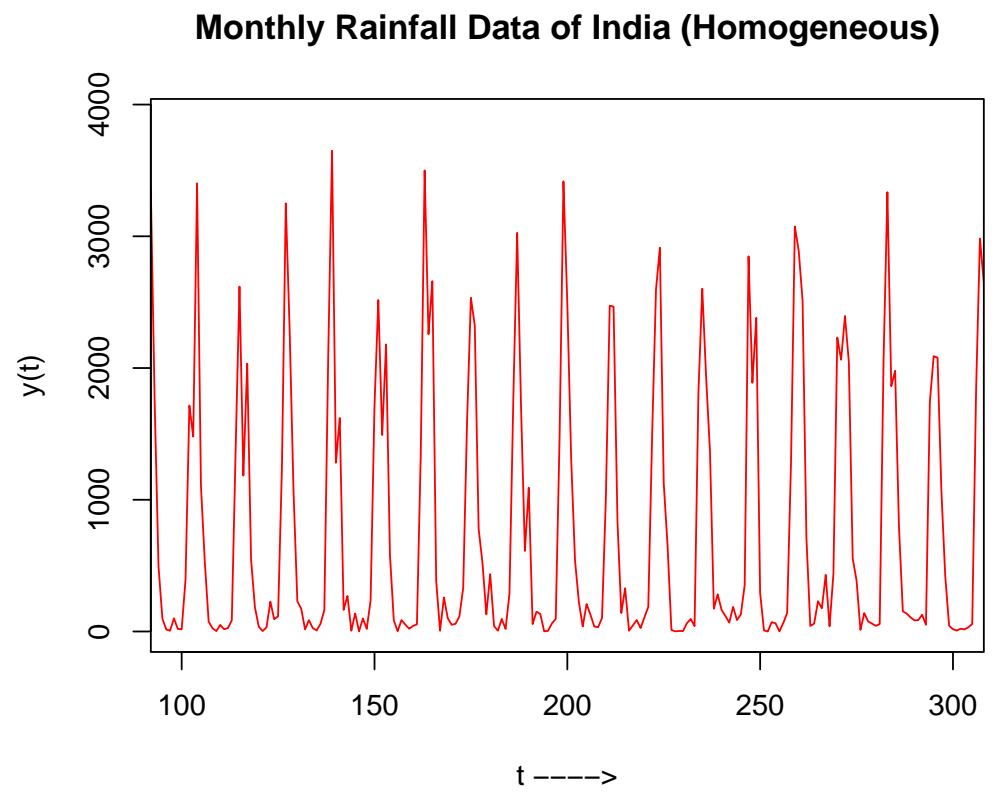

Figure 1: Homogeneous Indian Monthly Rainfall Data.

Fitted and Observed of Monthly Rainfall Data of India (Homogene

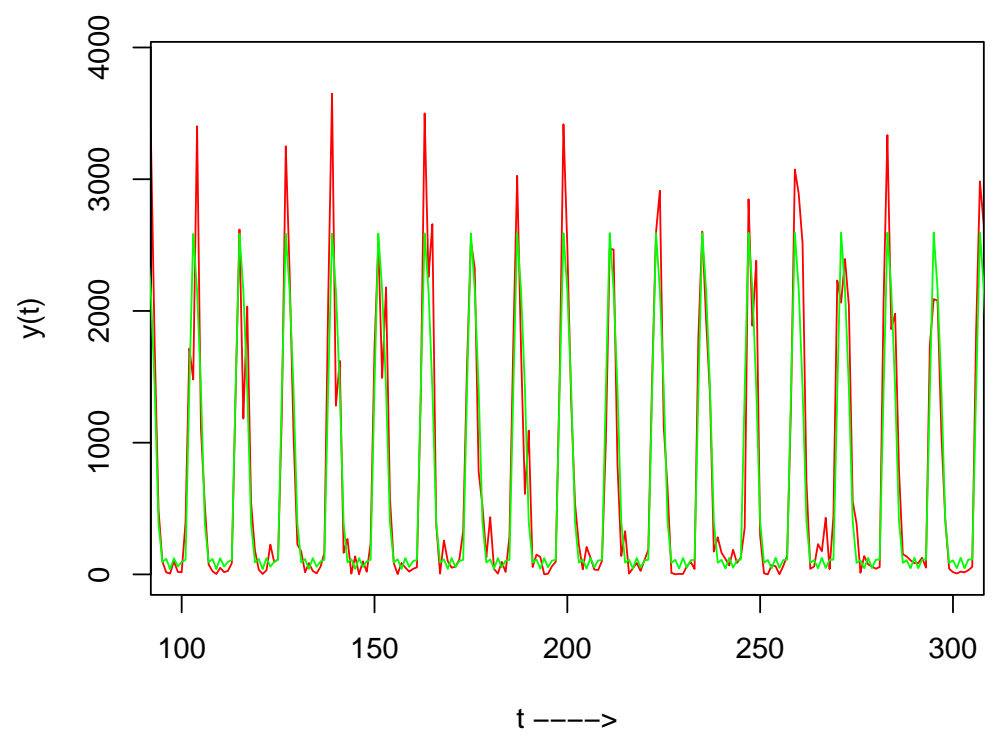

Figure 2: Fitted and Original Homogeneous Monthly Rainfall Data. 
of this model, but in this paper it is observed that if we use the Newton-Raphson method with proper step factor modification, the method works very well. Theoretically it is observed that the asymptotic variance of the proposed frequency estimator is smaller than the asymptotic variance of the corresponding LSE. The method can be easily used for multiple sinusoids also and it does not require higher dimensional optimization. It is observed in the numerical experiments that the MSEs of the proposed estimators are often less than the asymptotic variance of the LSEs. Since the iteration converges very quickly, it can be used quite effectively for on line implementation purpose.

\section{Appendix}

To prove this result, we use the following notation;

$$
D=\operatorname{diag}\{1,2, \ldots, n\}, \quad E=\left[\begin{array}{cc}
0 & 1 \\
-1 & 0
\end{array}\right], \quad \dot{X}=\frac{d}{d \omega} X=D X E, \quad \ddot{X}=\frac{d^{2}}{d \omega^{2}}=-D^{2} X .
$$

Note that $E E=-I, E E^{T}=I=E^{T} E$ and

$$
\frac{d}{d \omega}\left(X^{T} X\right)^{-1}=-\left(X^{T} X\right)^{-1}\left[\dot{X}^{T} X+X^{T} \dot{X}\right]\left(X^{T} X\right)^{-1} .
$$

Now we will compute $R^{\prime}(\omega)$ and $R^{\prime \prime}(\omega)$.

$$
\frac{1}{2} R^{\prime}(\omega)=Y^{T} \dot{X}\left(X^{T} X\right)^{-1} X^{T} Y-Y^{T} X\left(X^{T} X\right)^{-1} \dot{X}^{T} X\left(X^{T} X\right)^{-1} X^{T} Y .
$$

Similarly,

$$
\begin{aligned}
\frac{1}{2} R^{\prime \prime}(\omega) & =Y^{T} \ddot{X}\left(X^{T} X\right)^{-1} X^{T} Y-Y^{T} \dot{X}\left(X^{T} X\right)^{-1}\left(\dot{X}^{T} X+X^{T} \dot{X}\right)\left(X^{T} X\right)^{-1} X^{T} Y \\
& +Y^{T} \dot{X}\left(X^{T} X\right)^{-1} \dot{X}^{T} Y-Y^{T} \dot{X}\left(X^{T} X\right)^{-1} \dot{X}^{T} X\left(X^{T} X\right)^{-1} X^{T} Y \\
& +Y^{T} X\left(X^{T} X\right)^{-1}\left(\dot{X}^{T} X+X^{T} \dot{X}\right)\left(X^{T} X\right)^{-1} \dot{X}^{T} X\left(X^{T} X\right)^{-1} X^{T} Y \\
& -Y^{T} X\left(X^{T} X\right)^{-1}\left(\ddot{X}^{T} X\right)\left(X^{T} X\right)^{-1} X^{T} Y-Y^{T} X\left(X^{T} X\right)^{-1}\left(\dot{X}^{T} \dot{X}\right)\left(X^{T} X\right)^{-1} X^{T} Y \\
& +Y^{T} X\left(X^{T} X\right)^{-1} \dot{X}^{T} X\left(X^{T} X\right)^{-1}\left(\dot{X}^{T} X+X^{T} \dot{X}\right)\left(X^{T} X\right)^{-1} X^{T} Y \\
& -Y^{T} X\left(X^{T} X\right)^{-1} \dot{X}^{T} X\left(X^{T} X\right)^{-1} \dot{X}^{T} Y .
\end{aligned}
$$


Now we should like to approximate $\frac{1}{n^{3}} R^{\prime \prime}(\tilde{\omega})$ for large $n$. We are assuming that $\tilde{\omega}-\omega=$ $O_{p}\left(n^{-1-\delta}\right)$, therefore, for large $n,\left(\frac{1}{n} X(\tilde{\omega})^{T} X(\tilde{\omega})\right)^{-1}=2 I+O_{p}\left(\frac{1}{n}\right)$. Therefore, for large $n$,

$$
\begin{aligned}
\frac{1}{2 n^{3}} R^{\prime \prime}(\tilde{\omega}) & =\frac{2}{n^{4}} Y^{T} \ddot{X} X^{T} Y-\frac{4}{n^{5}} Y^{T} \dot{X}\left(\dot{X}^{T} X+X^{T} \dot{X}\right) X^{T} Y+\frac{2}{n^{4}} Y^{T} \dot{X} \dot{X}^{T} Y \\
& -\frac{4}{n^{5}} Y^{T} \dot{X} \dot{X}^{T} X X^{T} Y+\frac{8}{n^{6}} Y^{T} X\left(\dot{X}^{T} X+X^{T} \dot{X}\right) \dot{X}^{T} X X^{T} Y \\
& -\frac{4}{n^{5}} Y^{T} X \ddot{X}^{T} X X^{T} Y-\frac{4}{n^{5}} Y^{T} X \dot{X}^{T} \dot{X} X^{T} Y+\frac{8}{n^{6}} Y^{T} X \dot{X}^{T} X\left(\dot{X}^{T} X+X^{T} \dot{X}\right) X^{T} Y \\
& -\frac{4}{n^{5}} Y^{T} X \dot{X}^{T} X \dot{X}^{T} Y+O_{p}\left(\frac{1}{n}\right) .
\end{aligned}
$$

Substituting $\dot{X}$ and $\ddot{X}$ in terms of $D$ and $X$, we obtain

$$
\begin{aligned}
\frac{1}{2 n^{3}} R^{\prime \prime}(\tilde{\omega}) & =-\frac{2}{n^{4}} Y^{T} D^{2} X X^{T} Y-\frac{4}{n^{5}} Y^{T} D X E\left(E^{T} X^{T} D X+X^{T} D X E\right) X^{T} Y \\
& +\frac{2}{n^{4}} Y^{T} D X E E^{T} X^{T} D Y-\frac{4}{n^{5}} Y^{T} D X E E^{T} X^{T} D X X^{T} Y \\
& +\frac{8}{n^{6}} Y^{T} X\left(E^{T} X^{T} D X+X^{T} D X E\right) E^{T} X^{T} D X X^{T} Y+\frac{4}{n^{5}} Y^{T} X X^{T} D^{2} X X^{T} Y \\
& -\frac{4}{n^{5}} Y^{T} X E^{T} X^{T} D^{2} X E X^{T} Y+\frac{8}{n^{6}} Y^{T} X E^{T} X^{T} D X\left(E^{T} X^{T} D X+X^{T} D X E\right) X^{T} Y \\
& -\frac{4}{n^{5}} Y^{T} X E^{T} X^{T} D X E^{T} X^{T} D Y+O_{p}\left(\frac{1}{n}\right) .
\end{aligned}
$$

We will be using the following facts for $0<\omega<\pi$,

$$
\begin{array}{cc}
\sum_{t=1}^{n} t \cos ^{2}(\omega t)=\frac{n^{2}}{4}+O(n), & \sum_{t=1}^{n} t \sin ^{2}(\omega t)=\frac{n^{2}}{4}+O(n) . \\
\sum_{t=1}^{n} \cos ^{2}(\omega t)=\frac{n}{2}+o(n), & \sum_{t=1}^{n} \sin ^{2}(\omega t)=\frac{n}{2}+o(n) . \\
\sum_{t=1}^{n} t^{2} \cos ^{2}(\omega t)=\frac{n^{3}}{6}+O\left(n^{2}\right), & \sum_{t=1}^{n} t^{2} \sin ^{2}(\omega t)=\frac{n^{3}}{6}+O\left(n^{2}\right) .
\end{array}
$$

and

$$
\begin{gathered}
\frac{1}{n^{2}} Y^{T} D X=\frac{1}{4}\left(\begin{array}{ll}
A & B
\end{array}\right)+O_{p}\left(\frac{1}{n}\right), \quad \frac{1}{n^{3}} Y^{T} D^{2} X=\frac{1}{6}\left(\begin{array}{ll}
A & B
\end{array}\right)+O_{p}\left(\frac{1}{n}\right), \quad \frac{1}{n^{3}} X^{T} D^{2} X=\frac{1}{6} I+O_{p}\left(\frac{1}{n}\right) \\
\frac{1}{n} X^{T} Y=\frac{1}{2}(A B)^{T}+O_{p}\left(\frac{1}{n}\right), \quad \frac{1}{n^{2}} X^{T} D X=\frac{1}{4} I+O_{p}\left(\frac{1}{n}\right) \quad E E=-I, E E^{T}=I,
\end{gathered}
$$

Therefore,

$\frac{1}{2 n^{3}} R^{\prime \prime}(\tilde{\omega})=\left(A^{2}+B^{2}\right)\left[-\frac{1}{6}-0+\frac{1}{8}-\frac{1}{8}+0+\frac{1}{6}-\frac{1}{6}+0+\frac{1}{8}\right]+O_{p}\left(\frac{1}{n}\right)=-\frac{1}{24}\left(A^{2}+B^{2}\right)+O_{p}\left(\frac{1}{n}\right)$ 
Now we will consider the two terms of the numerator separately. First consider $\frac{1}{n^{3}} Y^{T} X\left(X^{T} X\right)^{-1} \dot{X}^{T} X\left(X^{T} X\right)^{-1} X^{T} Y$. Note that

$$
\begin{aligned}
\frac{1}{n^{3}} Y^{T} X\left(X^{T} X\right)^{-1} \dot{X}^{T} X\left(X^{T} X\right)^{-1} X^{T} Y & =\frac{1}{n^{3}} Y^{T} X\left(X^{T} X\right)^{-1} E^{T} X^{T} D X\left(X^{T} X\right)^{-1} X^{T} Y \\
& =\frac{1}{n^{3}} Y^{T} X\left(2 I+O_{p}\left(\frac{1}{n}\right)\right) E^{T}\left(\frac{1}{4} I+O_{p}\left(\frac{1}{n}\right)\right)\left(2 I+O_{p}\left(\frac{1}{n}\right)\right) X^{T} Y \\
& =\frac{1}{n^{3}} Y^{T} X E^{T} X^{T} Y+O_{p}\left(\frac{1}{n}\right)=O_{p}\left(\frac{1}{n}\right) .
\end{aligned}
$$

Note that $\frac{1}{n^{3}} Y^{T} \dot{X}\left(X^{T} X\right)^{-1} X^{T} Y=\frac{2}{n^{4}} Y^{T} D X E X^{T} Y$. Now we consider $Y^{T} D X E X^{T} Y$.

$$
\begin{aligned}
Y^{T} D X(\tilde{\omega}) E X^{T}(\tilde{\omega}) Y & =\left(\sum_{t=1}^{n} y(t) t \cos (\tilde{\omega} t)\right)\left(\sum_{t=1}^{n} y(t) \sin (\tilde{\omega} t)\right) \\
& -\left(\sum_{t=1}^{n} y(t) t \sin (\tilde{\omega} t)\right)\left(\sum_{t=1}^{n} y(t) \cos (\tilde{\omega} t)\right)
\end{aligned}
$$

If $\delta \in\left(0, \frac{1}{2}\right]$, then along the same line as Bai et al. [1] (see also Nandi and Kundu [8]), it can be shown that,

$$
\sum_{t=1}^{n} y(t) \cos (\tilde{\omega} t)=\frac{n}{2}\left(A+O_{p}\left(n^{-\delta}\right)\right), \quad \sum_{t=1}^{n} y(t) \sin (\tilde{\omega} t)=\frac{n}{2}\left(B+O_{p}\left(n^{-\delta}\right)\right) .
$$

Now if we consider;

$$
\begin{aligned}
\sum_{t=1}^{n} y(t) t e^{-i \tilde{\omega} t} & =\sum_{t=1}^{n}(A \cos (\omega t)+B \sin (\omega t)+X(t)) t e^{-i \tilde{\omega} t} \\
& =\frac{1}{2}(A-i B) \sum_{t=1}^{n} t e^{i(\omega-\tilde{\omega}) t}+\frac{1}{2}(A+i B) \sum_{t=1}^{n} t e^{-i(\omega+\tilde{\omega}) t}+\sum_{t=1}^{n} X(t) t e^{-i \tilde{\omega} t}
\end{aligned}
$$

It has been shown in Bai et al. [1] that

$$
\sum_{t=1}^{n} t e^{-i(\omega+\tilde{\omega}) t}=O_{p}(n)
$$

and

$$
\begin{gathered}
\sum_{t=1}^{n} t e^{i(\omega-\tilde{\omega}) t}=\sum_{t=1}^{n} t+i(\omega-\tilde{\omega}) \sum_{t=1}^{n} t^{2}-\frac{1}{2}(\omega-\tilde{\omega})^{2} \sum_{t=1}^{n} t^{3}-\frac{1}{6} i(\omega-\tilde{\omega})^{3} \sum_{t=1}^{n} t^{4}+ \\
\frac{1}{24}(\omega-\tilde{\omega})^{4} \sum_{t=1}^{n} t^{5} e^{i\left(\omega-\omega^{*}\right) t}
\end{gathered}
$$


Note that

$$
\frac{1}{24}(\omega-\tilde{\omega})^{4} \sum_{t=1}^{n} t^{5} e^{i\left(\omega-\omega^{*}\right) t}=O_{p}\left(n^{2-4 \delta}\right) .
$$

Choose $L$ large enough such that $L \delta>1$ and using the Taylor series expansion of $e^{-i \tilde{\omega} t}$ we obtain,

$$
\begin{aligned}
\sum_{t=1}^{n} X(t) t e^{-i \tilde{\omega} t}= & \sum_{k=-\infty}^{\infty} a(k) \sum_{t=1}^{n} e(t-k) t e^{-i \tilde{\omega} t} \\
= & \sum_{k=-\infty}^{\infty} a(k) \sum_{t=1}^{n} e(t-k) t e^{-i \omega t}+\sum_{k=-\infty}^{\infty} a(k) \sum_{l=1}^{L-1} \frac{(-i(\tilde{\omega}-\omega))^{l}}{l !} \sum_{t=1}^{n} e(t-k) t^{l+1} e^{-i \omega t} \\
& +\sum_{k=-\infty}^{\infty} a(k) \frac{\theta(n(\tilde{\omega}-\omega))^{L}}{L !} \sum_{t=1}^{n} t|e(t-k)|(\text { here }|\theta|<1) \\
= & \sum_{k=-\infty}^{\infty} a(k) \sum_{t=1}^{n} e(t-k) t e^{-i \omega t}+\sum_{l=1}^{L-1} O_{p}\left(n^{-(1+\delta) l}\right) O_{p}\left(n^{l+\frac{3}{2}}\right)+\sum_{k=-\infty}^{\infty} a(k) O_{p}\left(n^{\frac{5}{2}-L \delta}\right) \\
= & \sum_{k=-\infty}^{\infty} a(k) \sum_{t=1}^{n} e(t-k) t e^{-i \omega t}+O_{p}\left(n^{\frac{5}{2}-L \delta}\right)
\end{aligned}
$$

Therefore,

$$
\begin{aligned}
\sum_{t=1}^{n} y(t) t \cos (\tilde{\omega} t)= & \frac{1}{2}\left[A\left(\sum_{t=1}^{n} t-\frac{1}{2}(\omega-\tilde{\omega})^{2} \sum_{t=1}^{n} t^{3}\right)+B\left(\sum_{t=1}^{n}(\omega-\tilde{\omega}) t^{2}-\frac{1}{6}(\omega-\tilde{\omega})^{3} \sum_{t=1}^{n} t^{4}\right)\right] \\
& +\sum_{k=-\infty}^{\infty} a(k) \sum_{t=1}^{n} e(t-k) t \cos (\omega t)+O_{p}\left(n^{\frac{5}{2}-L \delta}\right)+O_{p}(n)+O_{p}\left(n^{2-4 \delta}\right) .
\end{aligned}
$$

Similarly,

$$
\begin{aligned}
\sum_{t=1}^{n} y(t) t \sin (\tilde{\omega} t)= & \frac{1}{2}\left[B\left(\sum_{t=1}^{n} t-\frac{1}{2}(\omega-\tilde{\omega})^{2} \sum_{t=1}^{n} t^{3}\right)-A\left(\sum_{t=1}^{n}(\omega-\tilde{\omega}) t^{2}-\frac{1}{6}(\omega-\tilde{\omega})^{3} \sum_{t=1}^{n} t^{4}\right)\right] \\
& +\sum_{k=-\infty}^{\infty} a(k) \sum_{t=1}^{n} e(t-k) t \sin (\omega t)+O_{p}\left(n^{\frac{5}{2}-L \delta}\right)+O_{p}(n)+O_{p}\left(n^{2-4 \delta}\right) .
\end{aligned}
$$


Therefore,

$$
\begin{aligned}
\widehat{\omega} & =\tilde{\omega}-\frac{1}{4} \frac{R^{\prime}(\tilde{\omega})}{R^{\prime \prime}(\tilde{\omega})} \\
& =\tilde{\omega}-\frac{1}{4} \frac{\frac{1}{2 n^{3}} R^{\prime}(\tilde{\omega})}{-\frac{1}{24}\left(A^{2}+B^{2}\right)+O_{p}\left(\frac{1}{n}\right)} \\
& =\tilde{\omega}-\frac{1}{4} \frac{\frac{2}{n^{4}} Y^{T} D X E X^{T} Y}{-\frac{1}{24}\left(A^{2}+B^{2}\right)+O_{p}\left(\frac{1}{n}\right)} \\
& =\tilde{\omega}+12 \frac{\frac{1}{n^{4}} Y^{T} D X E X^{T} Y}{\left(A^{2}+B^{2}\right)+O_{p}\left(\frac{1}{n}\right)} \\
& =\tilde{\omega}+12 \frac{\frac{1}{4 n^{3}}\left(A^{2}+B^{2}\right)\left\{(\omega-\tilde{\omega}) \sum_{t=1}^{n} t^{2}-\frac{1}{6}(\omega-\tilde{\omega})^{3} \sum_{t=1}^{n} t^{4}\right\}}{\left(A^{2}+B^{2}\right)+O_{p}\left(\frac{1}{n}\right)} \\
& +\frac{6}{\left(A^{2}+B^{2}\right) n^{3}+O_{p}\left(\frac{1}{n}\right)}\left[B \sum_{k=-\infty}^{\infty} a(k) \sum_{t=1}^{n} e(t-k) t \cos (\omega t)+A \sum_{k=-\infty}^{\infty} a(k) \sum_{t=1}^{n} e(t-k) t \sin (\omega t)\right] \\
+ & O_{p}\left(n^{-\frac{1}{2}-L \delta}\right)+O_{p}\left(n^{-2}\right)+O_{p}\left(n^{-1-4 \delta}\right) \\
& =\omega+(\omega-\tilde{\omega}) O_{p}\left(n^{-2 \delta}\right) \\
& +\frac{6}{\left(A^{2}+B^{2}\right) n^{3}+O_{p}\left(\frac{1}{n}\right)}\left[B \sum_{k=-\infty}^{\infty} a(k) \sum_{t=1}^{n} e(t-k) t \cos (\omega t)+A \sum_{k=-\infty}^{\infty} a(k) \sum_{t=1}^{n} e(t-k) t \sin (\omega t)\right] \\
+ & O_{p}\left(n^{-\frac{1}{2}-L \delta}\right)+O_{p}\left(n^{-2}\right)+O_{p}\left(n^{-1-4 \delta}\right)
\end{aligned}
$$

Therefore, if $\delta \leq \frac{1}{6}$, then clearly $\widehat{\omega}-\omega=O_{p}\left(n^{-1-3 \delta}\right)$, and if $\delta>\frac{1}{6}$, then

$$
\begin{aligned}
n^{\frac{3}{2}}(\widehat{\omega}-\omega) & \stackrel{d}{=} \frac{6 n^{-\frac{3}{2}}}{\left(A^{2}+B^{2}\right)}\left[B \sum_{k=-\infty}^{\infty} a(k) \sum_{t=1}^{n} e(t-k) t \cos (\omega t)+A \sum_{k=-\infty}^{\infty} a(k) \sum_{t=1}^{n} e(t-k) t \sin (\omega t)\right] \\
& =\frac{6 n^{-\frac{3}{2}}}{\left(A^{2}+B^{2}\right)}\left[B \sum_{t=1}^{n} X(t) e(t-k) t \cos (\omega t)+A \sum_{t=1}^{n} X(t) e(t-k) t \sin (\omega t)\right] \\
& \stackrel{d}{\longrightarrow} N\left(0, \frac{6 \sigma^{2} c}{A^{2}+B^{2}}\right) .
\end{aligned}
$$

\section{References}

[1] Bai, Z.D., Rao, C.R., Chow, M. and Kundu, D. (2003), "An efficient algorithm for estimating the parameters of superimposed exponential signals", Journal of Statistical Planning and Inference, 110, 23 - 34. 
[2] Bai, Z.D, Rao, C.R., Wu, Y., Zen, M-M.,; Zhao, L.C. (1999), "The simultaneous estimation of the number of signals and frequencies of multiple sinusoids when some observations are missing. I. Asymptotic", Proceedings of the National Academy of Sciences USA, 96, 11106-11110.

[3] Bresler, Y. and Macovski (1988), "Exact maximum likelihood parameter estimation of superimposed exponential signals in noise", IEEE Transactions on Acoustics Speech and Signal Processing, 34, 1081-1089.

[4] Chan, Y. T., Lavoie, J. M. M. and Plant, J. B. (1981), "A parameter estimation approach to estimation of frequencies of sinusoids", IEEE Trans. Acoust. Speech and Signal Processing, ASSP-29, 214-219.

[5] Fisher, R.A. (1929) "Tests of significance in Harmonic analysis", Proc. Royal Soc. London, Ser A, 125, 54-59.

[6] Hannan, E.J. (1973), "Estimation of frequencies", Journal of Applied Probability, 10, 510-519.

[7] Kumaresan, R., Scharf, L. L. and Shaw, A. K. (1986), "An algorithm for poll-zero modeling and spectral analysis", IEEE Transaction on Acoustics Speech and Signal Processing, 34, 637-640.

[8] Nandi, S. and Kundu, D. (2006), "A fast and efficient algorithm for estimating the parameters of sum of sinusoidal model", Sankhya, 68, 283-306.

[9] Pisarenko, V. F. (1973), "The retrieval of harmonics from a covariance function", Jour. Royal Astr. Soc., 33, 347-366.

[10] Press, W. H., Teukolsky, S. A., Vetterling, W. T., Flannery, B. P., (1993), Numerical Recipes in FORTRAN, Cambridge University Press, Cambridge.

[11] Rice, J. A. and Rosenblatt, M. (1988), "On frequency estimation", Biometrika, 75, $477-484$. 
[12] Richards, F. S. G. (1961), "A method of maximum likelihood estimation", Journal of the Royal Statistical Society B, 23, 469-475.

[13] Smyth, G. K. (2000) “Employing symmetry constraints for improved frequency estimation by eigenanalysis methods", Technometrics, 42, 277-289.

[14] Song, K.S. and Li, T.H. (2006), "On convergence and bias correction of a joint estimation algorithm for multiple sinusoidal frequencies", Journal of the American Statistical Association, 101, $830-842$.

[15] Stoica, P. (1993), "List of references on spectral line analysis", Signal Processing, 31, 329-340.

[16] Walker, A.M. (1971), "On the estimation of a Harmonic components in a time series with stationary residuals", Biometrika, 58, 21-36. 
Table 2: Model 1: The average estimates (AEs), the mean squared errors (MSEs), the asymptotic variances of the LSE (ASVs) and the asymptotic variances of the modified Newton-Raphson method (ASVPs) of $\widehat{\omega}$, are reported.

\begin{tabular}{|c|c|c|c|c|c|c|c|}
\hline & & $N=100$ & $N=200$ & $N=300$ & $N=400$ & $N=500$ & $N=1000$ \\
\hline \multirow{4}{*}{$\sigma=.25$} & $\mathrm{AE}$ & .499987 & .499995 & .499998 & 499999 & 499999 & 0.500000 \\
\hline & MSE & $6.93174 \mathrm{e}-7$ & $9.04893 \mathrm{e}-8$ & $2.61876 \mathrm{e}-8$ & $1.13173 \mathrm{e}-8$ & $5.75471 \mathrm{e}-9$ & $5.27773 \mathrm{e}-10$ \\
\hline & ASV & $1.07958 \mathrm{e}-6$ & $1.34947 \mathrm{e}-7$ & $3.99844 \mathrm{e}-8$ & $1.68684 \mathrm{e}-8$ & $8.63662 \mathrm{e}-9$ & $1.07958 \mathrm{e}-9$ \\
\hline & ASVP & $2.69894 \mathrm{e}-7$ & $3.37368 \mathrm{e}-8$ & $9.99609 \mathrm{e}-9$ & $4.21710 \mathrm{e}-9$ & $2.15916 \mathrm{e}-9$ & $2.69894 \mathrm{e}-10$ \\
\hline \multirow{4}{*}{$\sigma=.50$} & $\mathrm{AE}$ & .499964 & .499988 & 0.499997 & .499998 & .499997 & .500000 \\
\hline & MSE & $2.78071 \mathrm{e}-6$ & $3.62227 \mathrm{e}-7$ & $1.04880 \mathrm{e}-7$ & $4.52979 \mathrm{e}-8$ & $2.30361 \mathrm{e}-8$ & $2.11131 \mathrm{e}-9$ \\
\hline & $\mathrm{ASV}$ & $4.31831 \mathrm{e}-6$ & $5.39789 \mathrm{e}-7$ & $1.59937 \mathrm{e}-7$ & $6.74736 \mathrm{e}-8$ & $3.45465 \mathrm{e}-8$ & $4.31831 \mathrm{e}-9$ \\
\hline & ASVP & $1.07958 \mathrm{e}-6$ & $1.34947 \mathrm{e}-7$ & $3.99844 \mathrm{e}-8$ & $1.68684 \mathrm{e}-8$ & $8.63662 \mathrm{e}-9$ & $1.07958 \mathrm{e}-9$ \\
\hline \multirow{4}{*}{$\sigma=.75$} & $\mathrm{AE}$ & .499930 & .499980 & .499995 & .499996 & .499996 & .500000 \\
\hline & MSE & $6.30149 \mathrm{e}-6$ & $8.17229 \mathrm{e}-7$ & $2.36489 \mathrm{e}-7$ & $1.02050 \mathrm{e}-7$ & $5.19003 \mathrm{e}-8$ & $4.75294 \mathrm{e}-9$ \\
\hline & ASV & $9.71620 \mathrm{e}-6$ & $1.21452 \mathrm{e}-6$ & $3.59859 \mathrm{e}-7$ & $1.51816 \mathrm{e}-7$ & $7.77296 \mathrm{e}-8$ & $9.71620 \mathrm{e}-9$ \\
\hline & ASVP & $2.42905 \mathrm{e}-6$ & $3.03631 \mathrm{e}-7$ & $8.99648 \mathrm{e}-8$ & $3.79539 \mathrm{e}-8$ & $1.94324 \mathrm{e}-8$ & $2.42905 \mathrm{e}-9$ \\
\hline \multirow{4}{*}{$\sigma=1.0$} & $\mathrm{AE}$ & .499885 & .499971 & .499993 & .499994 & .499994 & .499999 \\
\hline & MSE & $1.13361 \mathrm{e}-5$ & $1.45972 \mathrm{e}-6$ & $4.21734 \mathrm{e}-7$ & $1.81773 \mathrm{e}-7$ & $9.24465 \mathrm{e}-8$ & $8.45772 \mathrm{e}-9$ \\
\hline & $\mathrm{ASV}$ & $1.72732 \mathrm{e}-5$ & $2.15916 \mathrm{e}-6$ & $6.39750 \mathrm{e}-7$ & $2.69894 \mathrm{e}-7$ & $1.38186 \mathrm{e}-7$ & $1.72732 \mathrm{e}-8$ \\
\hline & ASVP & $4.31831 \mathrm{e}-6$ & $5.39789 \mathrm{e}-7$ & $1.59937 \mathrm{e}-7$ & $6.74736 \mathrm{e}-8$ & $3.45465 \mathrm{e}-8$ & $4.31831 \mathrm{e}-9$ \\
\hline \multirow{4}{*}{$\sigma=1.5$} & $\mathrm{AE}$ & .499761 & .499948 & .499989 & .499989 & .499990 & .499999 \\
\hline & MSE & $2.65572 \mathrm{e}-5$ & $3.33704 \mathrm{e}-6$ & $9.57806 \mathrm{e}-7$ & $4.11432 \mathrm{e}-7$ & $2.09206 \mathrm{e}-7$ & $1.90909 \mathrm{e}-8$ \\
\hline & $\mathrm{ASV}$ & $3.88648 \mathrm{e}-5$ & $4.85810 \mathrm{e}-6$ & $1.43944 \mathrm{e}-6$ & $6.07262 \mathrm{e}-7$ & $3.10918 \mathrm{e}-7$ & $3.88648 \mathrm{e}-8$ \\
\hline & ASVP & $9.71620 \mathrm{e}-6$ & $1.21452 \mathrm{e}-6$ & $3.59859 \mathrm{e}-7$ & $1.51816 \mathrm{e}-7$ & $7.77296 \mathrm{e}-8$ & $9.71620 \mathrm{e}-9$ \\
\hline
\end{tabular}


Table 3: Model 2: The average estimates (AEs), the mean squared errors (MSEs), the asymptotic variances of the LSE (ASVs) and the asymptotic variances of the modified Newton-Raphson method (ASVPs) of $\widehat{\omega}_{1}$, are reported.

\begin{tabular}{|c|l|c|c|c|c|c|c|}
\hline \multirow{5}{*}{$\sigma=.25$} & & $N=100$ & $N=200$ & $N=300$ & $N=400$ & $N=500$ & $N=1000$ \\
\hline & AE & 1.500656 & 1.500252 & 1.500099 & 1.500019 & 1.499985 & 1.500000 \\
\cline { 2 - 8 } & MSE & $6.95725-7$ & $9.87144 \mathrm{e}-8$ & $1.87342 \mathrm{e}-8$ & $4.21747 \mathrm{e}-9$ & $2.28201 \mathrm{e}-9$ & $2.46321 \mathrm{e}-10$ \\
\cline { 2 - 8 } & ASV & $6.25727 \mathrm{e}-7$ & $7.82159-8$ & $2.31751 \mathrm{e}-8$ & $9.77699 \mathrm{e}-9$ & $5.00582 \mathrm{e}-9$ & $6.25727 \mathrm{e}-10$ \\
\cline { 2 - 8 } & ASVP & $1.56432 \mathrm{e}-7$ & $1.95540 \mathrm{e}-8$ & $5.79377 \mathrm{e}-9$ & $2.44425 \mathrm{e}-9$ & $1.25145 \mathrm{e}-9$ & $1.56432 \mathrm{e}-10$ \\
\hline \multirow{5}{*}{$\sigma=.50$} & AE & 1.500648 & 1.500253 & 1.500100 & 1.500019 & 1.499985 & 1.500000 \\
\cline { 2 - 8 } & MSE & $1.48463 \mathrm{e}-6$ & $2.05632 \mathrm{e}-7$ & $4.56039 \mathrm{e}-8$ & $1.57820 \mathrm{e}-8$ & $8.46078 \mathrm{e}-9$ & $9.85326 \mathrm{e}-10$ \\
\cline { 2 - 8 } & ASV & $2.50291 \mathrm{e}-6$ & $3.12864 \mathrm{e}-7$ & $9.27003 \mathrm{e}-8$ & $3.91079 \mathrm{e}-8$ & $2.00232 \mathrm{e}-8$ & $2.50291 \mathrm{e}-9$ \\
\cline { 2 - 8 } & ASVP & $6.25727 \mathrm{e}-7$ & $7.82159 \mathrm{e}-8$ & $2.31751 \mathrm{e}-8$ & $9.77699 \mathrm{e}-9$ & $5.00582 \mathrm{e}-9$ & $6.25727 \mathrm{e}-10$ \\
\hline \multirow{5}{*}{$\sigma=.75$} & AE & 1.500639 & 1.500254 & 1.500100 & 1.500019 & 1.499985 & 1.500000 \\
\cline { 2 - 8 } & MSE & $2.81215 \mathrm{e}-6$ & $3.83646 \mathrm{e}-7$ & $9.03823 \mathrm{e}-8$ & $3.50692 \mathrm{e}-8$ & $1.87605 \mathrm{e}-8$ & $2.21783 \mathrm{e}-9$ \\
\cline { 2 - 8 } & ASV & $5.63154 \mathrm{e}-6$ & $7.03943 \mathrm{e}-7$ & $2.08576 \mathrm{e}-7$ & $8.79929 \mathrm{e}-8$ & $4.50524 \mathrm{e}-8$ & $5.63154 \mathrm{e}-9$ \\
\cline { 2 - 8 } & ASVP & $1.40789 \mathrm{e}-6$ & $1.75986 \mathrm{e}-7$ & $5.21439 \mathrm{e}-8$ & $2.19982 \mathrm{e}-8$ & $1.12631 \mathrm{e}-8$ & $1.40789 \mathrm{e}-9$ \\
\hline \multirow{5}{*}{$\sigma=1.0$} & AE & 1.500628 & 1.500254 & 1.500101 & 1.500018 & 1.499985 & 1.500000 \\
\cline { 2 - 8 } & MSE & $4.68938 \mathrm{e}-6$ & $6.33231 \mathrm{e}-7$ & $1.53175 \mathrm{e}-7$ & $6.21053 \mathrm{e}-8$ & $3.31897 \mathrm{e}-8$ & $3.94492 \mathrm{e}-9$ \\
\cline { 2 - 8 } & ASV & $1.00116 \mathrm{e}-5$ & $1.25145 \mathrm{e}-6$ & $3.70801 \mathrm{e}-7$ & $1.56432 \mathrm{e}-7$ & $8.00931 \mathrm{e}-8$ & $1.00116 \mathrm{e}-8$ \\
\cline { 2 - 8 } & ASVP & $2.50291 \mathrm{e}-6$ & $3.12864 \mathrm{e}-7$ & $9.27003 \mathrm{e}-8$ & $3.91079 \mathrm{e}-8$ & $2.00233 \mathrm{e}-8$ & $2.50291 \mathrm{e}-9$ \\
\hline & AE & 1.500601 & 1.500255 & 1.500102 & 1.500018 & 1.499984 & 1.500000 \\
\cline { 2 - 7 } & MSE & $1.01610 \mathrm{e}-5$ & $1.35033 \mathrm{e}-6$ & $3.33528 \mathrm{e}-7$ & $1.39621 \mathrm{e}-7$ & $7.44919 \mathrm{e}-8$ & $8.88922 \mathrm{e}-9$ \\
\cline { 2 - 7 } & ASV & $2.25262 \mathrm{e}-5$ & $2.81577 \mathrm{e}-6$ & $8.34303 \mathrm{e}-7$ & $3.51972 \mathrm{e}-7$ & $1.80209 \mathrm{e}-7$ & $2.25262 \mathrm{e}-8$ \\
\hline \multirow{5}{*}{$=1.5$} & $5.63154 \mathrm{e}-6$ & $7.03943 \mathrm{e}-7$ & $2.08576 \mathrm{e}-7$ & $8.79929 \mathrm{e}-8$ & $4.50524 \mathrm{e}-8$ & $5.63154 \mathrm{e}-9$ \\
\hline
\end{tabular}


Table 4: Model 2: The average estimates (AEs), the mean squared errors (MSEs), the asymptotic variances of the LSE (ASVs) and the asymptotic variances of the modified Newton-Raphson method (ASVPs) of $\widehat{\omega}_{2}$, are reported.

\begin{tabular}{|l|l|c|c|c|c|c|c|}
\hline \multirow{5}{*}{$\sigma=.25$} & & $N=100$ & $N=200$ & $N=300$ & $N=400$ & $N=500$ & $N=1000$ \\
\hline & AE & .500001 & .499998 & 0.499999 & .499999 & .499999 & .500000 \\
\cline { 2 - 8 } & MSE & $6.94534 \mathrm{e}-7$ & $9.04881 \mathrm{e}-8$ & $2.62034 \mathrm{e}-8$ & $1.13147 \mathrm{e}-8$ & $5.75764 \mathrm{e}-9$ & $5.27798 \mathrm{e}-10$ \\
\cline { 2 - 8 } & ASV & $1.07958 \mathrm{e}-6$ & $1.34947 \mathrm{e}-7$ & $3.99844 \mathrm{e}-8$ & $1.68684 \mathrm{e}-8$ & $8.63662 \mathrm{e}-9$ & $1.07958 \mathrm{e}-9$ \\
\cline { 2 - 8 } & ASVP & $2.69894 \mathrm{e}-7$ & $3.37368 \mathrm{e}-8$ & $9.99609 \mathrm{e}-9$ & $4.21710 \mathrm{e}-9$ & $2.15916 \mathrm{e}-9$ & $2.69894 \mathrm{e}-10$ \\
\hline \multirow{5}{*}{$\sigma=.50$} & AE & .499976 & .499991 & 0.499997 & .499998 & .499997 & .500000 \\
\cline { 2 - 8 } & MSE & $2.78543 \mathrm{e}-6$ & $3.62252 \mathrm{e}-7$ & $1.04944 \mathrm{e}-7$ & $4.52863 \mathrm{e}-8$ & $2.30464 \mathrm{e}-8$ & $2.11141 \mathrm{e}-9$ \\
\cline { 2 - 8 } & ASV & $4.31831 \mathrm{e}-6$ & $5.39789 \mathrm{e}-7$ & $1.59937 \mathrm{e}-7$ & $6.74736 \mathrm{e}-8$ & $3.45465 \mathrm{e}-8$ & $4.31831 \mathrm{e}-9$ \\
\cline { 2 - 8 } & ASVP & $1.07958 \mathrm{e}-6$ & $1.34947 \mathrm{e}-7$ & $3.99844 \mathrm{e}-8$ & $1.68684 \mathrm{e}-8$ & $8.63662 \mathrm{e}-9$ & $1.07958 \mathrm{e}-9$ \\
\hline \multirow{5}{*}{$\sigma=.75$} & AE & .499940 & .499983 & .499995 & .499996 & .499996 & .500000 \\
\cline { 2 - 8 } & MSE & $6.31046 \mathrm{e}-6$ & $8.17308 \mathrm{e}-7$ & $2.36631 \mathrm{e}-7$ & $1.02023 \mathrm{e}-7$ & $5.19224 \mathrm{e}-8$ & $4.75317 \mathrm{e}-9$ \\
\cline { 2 - 8 } & ASV & $9.71620 \mathrm{e}-6$ & $1.21452 \mathrm{e}-6$ & $3.59859 \mathrm{e}-7$ & $1.51816 \mathrm{e}-7$ & $7.77296 \mathrm{e}-8$ & $9.71620 \mathrm{e}-9$ \\
\cline { 2 - 8 } & ASVP & $2.42905 \mathrm{e}-6$ & $3.03631 \mathrm{e}-7$ & $8.99648 \mathrm{e}-8$ & $3.79539 \mathrm{e}-8$ & $1.94324 \mathrm{e}-8$ & $2.42905 \mathrm{e}-9$ \\
\hline \multirow{5}{*}{$\sigma=1.0$} & AE & .499892 & .499974 & .499993 & .499994 & .499994 & .499999 \\
\cline { 2 - 8 } & MSE & $1.13484 \mathrm{e}-5$ & $1.45986 \mathrm{e}-6$ & $4.21975 \mathrm{e}-7$ & $1.81723 \mathrm{e}-7$ & $9.24846 \mathrm{e}-8$ & $8.45813 \mathrm{e}-9$ \\
\cline { 2 - 8 } & ASV & $1.72732 \mathrm{e}-5$ & $2.15916 \mathrm{e}-6$ & $6.39750 \mathrm{e}-7$ & $2.69894 \mathrm{e}-7$ & $1.38186 \mathrm{e}-7$ & $1.72732 \mathrm{e}-8$ \\
\cline { 2 - 8 } & ASVP & $4.31831 \mathrm{e}-6$ & $5.39789 \mathrm{e}-7$ & $1.59937 \mathrm{e}-7$ & $6.74736 \mathrm{e}-8$ & $3.45465 \mathrm{e}-8$ & $4.31831 \mathrm{e}-9$ \\
\hline & AE & .499761 & .499950 & .499990 & .499988 & .499990 & .499999 \\
\cline { 2 - 7 } & MSE & $2.65636 \mathrm{e}-5$ & $3.33721 \mathrm{e}-6$ & $9.58283 \mathrm{e}-7$ & $4.11319 \mathrm{e}-7$ & $2.09287 \mathrm{e}-7$ & $1.90919 \mathrm{e}-8$ \\
\cline { 2 - 7 } & ASV & $3.88648 \mathrm{e}-5$ & $4.85810 \mathrm{e}-6$ & $1.43944 \mathrm{e}-6$ & $6.07262 \mathrm{e}-7$ & $3.10918 \mathrm{e}-7$ & $3.88648 \mathrm{e}-8$ \\
\hline \multirow{5}{*}{} & $9.71620 \mathrm{e}-6$ & $1.21452 \mathrm{e}-6$ & $3.59859 \mathrm{e}-7$ & $1.51816 \mathrm{e}-7$ & $7.77296 \mathrm{e}-8$ & $9.71620 \mathrm{e}-9$ \\
\hline
\end{tabular}


Table 5: Model 1: The average estimates (AEs), the mean squared errors (MSEs), the asymptotic variances of the LSE (ASVs) and the asymptotic variances of the modified Newton-Raphson method (ASVPs) of $\widehat{A}$ are reported.

\begin{tabular}{|c|c|c|c|c|c|c|c|}
\hline & & $N=100$ & $N=200$ & $N=300$ & $N=400$ & $N=500$ & $N=1000$ \\
\hline \multirow{4}{*}{$\sigma=.25$} & $\mathrm{AE}$ & 1.412549 & 1.413092 & 1.413487 & 1.413667 & 1.413661 & 1.414106 \\
\hline & MSE & $6.12535 \mathrm{e}-3$ & $2.84327 \mathrm{e}-3$ & $1.61372 \mathrm{e}-3$ & $1.13530 \mathrm{e}-3$ & $8.45459 \mathrm{e}-4$ & $3.56515 \mathrm{e}-4$ \\
\hline & ASV & $8.99648 \mathrm{e}-3$ & $4.49824 \mathrm{e}-3$ & $2.99883 \mathrm{e}-3$ & $2.24912 \mathrm{e}-3$ & $1.79930 \mathrm{e}-3$ & $8.99648 \mathrm{e}-4$ \\
\hline & ASVP & $2.24912 \mathrm{e}-3$ & $1.12456 \mathrm{e}-3$ & $7.49707 \mathrm{e}-4$ & $5.62280 \mathrm{e}-4$ & $4.49824 \mathrm{e}-4$ & $2.24912 \mathrm{e}-4$ \\
\hline \multirow{4}{*}{$\sigma=.50$} & $\mathrm{AE}$ & 1.409042 & 1.410975 & 1.412144 & 1.412514 & 1.412689 & 1.413845 \\
\hline & MSE & $2.45701 \mathrm{e}-2$ & $1.14039 \mathrm{e}-2$ & $6.46766 \mathrm{e}-3$ & $4.55338 \mathrm{e}-3$ & $3.38801 \mathrm{e}-3$ & $1.42687 \mathrm{e}-3$ \\
\hline & ASV & $3.59859 \mathrm{e}-2$ & $1.79930 \mathrm{e}-2$ & $1.19953 \mathrm{e}-2$ & $8.99648 \mathrm{e}-3$ & 7.19718e-3 & $3.59859 \mathrm{e}-3$ \\
\hline & ASVP & $8.99648 \mathrm{e}-3$ & $4.49824 \mathrm{e}-3$ & $2.99883 \mathrm{e}-3$ & $2.24912 \mathrm{e}-3$ & $1.79930 \mathrm{e}-3$ & $8.99648 \mathrm{e}-4$ \\
\hline \multirow{4}{*}{$\sigma=.75$} & $\mathrm{AE}$ & 1.403658 & 1.407859 & 1.410182 & 1.410756 & 1.411298 & 1.413430 \\
\hline & MSE & $5.55551 \mathrm{e}-2$ & $2.57668 \mathrm{e}-2$ & $1.45985 \mathrm{e}-2$ & $1.02875 \mathrm{e}-2$ & $7.64361 \mathrm{e}-3$ & $3.21326 \mathrm{e}-3$ \\
\hline & ASV & $8.09683 \mathrm{e}-2$ & $4.04842 \mathrm{e}-2$ & $2.69894 \mathrm{e}-2$ & $2.02421 \mathrm{e}-2$ & $1.61937 \mathrm{e}-2$ & $8.09683 \mathrm{e}-3$ \\
\hline & ASVP & $2.02421 \mathrm{e}-2$ & $1.01210 \mathrm{e}-2$ & $6.74736 \mathrm{e}-3$ & $5.06052 \mathrm{e}-3$ & $4.04842 \mathrm{e}-3$ & $2.02421 \mathrm{e}-3$ \\
\hline \multirow{4}{*}{$\sigma=1.0$} & $\mathrm{AE}$ & 1.396328 & 1.403726 & 1.407597 & 1.408392 & 1.409486 & 1.412864 \\
\hline & MSE & $9.94778 \mathrm{e}-2$ & $4.60689 \mathrm{e}-2$ & $2.60666 \mathrm{e}-2$ & $1.83907 \mathrm{e}-2$ & $1.36374 \mathrm{e}-2$ & $5.71919 \mathrm{e}-2$ \\
\hline & ASV & .143944 & $7.19718 \mathrm{e}-2$ & $4.79812 \mathrm{e}-2$ & $3.59859 \mathrm{e}-2$ & $2.87887 \mathrm{e}-2$ & $1.43944 \mathrm{e}-2$ \\
\hline & ASVP & $3.59859 \mathrm{e}-2$ & $1.79930 \mathrm{e}-2$ & $1.19953 \mathrm{e}-2$ & $8.99648 \mathrm{e}-3$ & $7.19718 \mathrm{e}-3$ & $3.59859 \mathrm{e}-3$ \\
\hline \multirow{4}{*}{$\sigma=1.5$} & $\mathrm{AE}$ & 1.375450 & 1.392299 & 1.400515 & 1.401840 & 1.404590 & 1.411270 \\
\hline & MSE & .228936 & .105319 & $5.93737 \mathrm{e}-2$ & $4.20142 \mathrm{e}-2$ & $3.09841 \mathrm{e}-2$ & $1.29102 \mathrm{e}-2$ \\
\hline & ASV & .323873 & .161937 & .107958 & $8.09683 \mathrm{e}-2$ & $6.47747 \mathrm{e}-2$ & $3.23873 \mathrm{e}-2$ \\
\hline & ASVP & $8.09683 \mathrm{e}-2$ & $4.04842 \mathrm{e}-2$ & $2.69894 \mathrm{e}-2$ & $2.02421 \mathrm{e}-2$ & $1.61937 \mathrm{e}-2$ & $8.09683 \mathrm{e}-3$ \\
\hline
\end{tabular}

